\title{
Diagnostic Yield of Catheter Angiography in Patients with Subarachnoid Hemorrhage and Negative Initial Noninvasive Neurovascular Examinations
}

\author{
J.E. Delgado Almandoz, B.M. Crandall, J.L. Fease, J.M. Scholz, R.E. Anderson, Y. Kadkhodayan, and D.E. Tubman
}

\begin{abstract}
BACKGROUND AND PURPOSE: The yield of DSA in patients with SAH and negative initial noninvasive neurovascular examinations (CTA or MRA) is not well-understood. This study aimed to determine the yield of DSA for the detection of causative vascular lesions in this clinical scenario.
\end{abstract}

MATERIALS AND METHODS: We examined the yield of DSA for the detection of causative vascular lesions in a cohort of patients presenting to our institution with SAH and negative initial noninvasive neurovascular examinations during a 5-year period. Two experienced neuroradiologists independently evaluated the NCCT to determine the SAH pattern (diffuse, perimesencephalic, or peripheral sulcal) and the catheter angiograms to assess the presence of a causative vascular lesion.

RESULTS: Fifty-five patients were included in the study, with a mean age of 58.2 years (median, 58 years; range, $25-88$ years). Twenty-eight patients were men (50.9\%), and 27 were women (49.1\%). The initial noninvasive examination was a CTA in 47 patients (85.5\%) and an MRA in 8 patients (14.5\%). Thirty-three patients had diffuse SAH (60\%); 11, perimesencephalic SAH (20\%); and 11, peripheral sulcal SAH (20\%). DSA demonstrated a causative vascular lesion in 6 patients (10.9\%), 5 of whom had diffuse SAH (yield of 15.2\%) and 1 of whom had peripheral sulcal SAH (yield of 9.1\%). No causative vascular lesions were found in patients with perimesencephalic SAH.

CONCLUSIONS: DSA is a valuable tool in the evaluation of patients with diffuse and peripheral sulcal SAH who have negative initial noninvasive neurovascular examinations, demonstrating a causative vascular lesion in $15.2 \%$ and $9.1 \%$ of patients, respectively.

ABBREVIATIONS: $\mathrm{Cl}=$ confidence interval; DAVF, dural arteriovenous fistula

$\mathbf{N}$ ontraumatic SAH affects approximately 20,000-30,000 people in the United States each year ${ }^{1}$ and carries up to $45 \%$ thirty-day mortality as well as poor functional outcome among survivors. ${ }^{2-5}$ Although most cases of SAH are caused by ruptured cerebral aneurysms, ${ }^{4}$ prior studies have shown that in $5 \%-36 \%$ of cases, the initial neurovascular examination does not reveal a causative cerebral aneurysm or other vascular abnormality. ${ }^{6-31}$ Prompt identification of patients who have a vascular abnor-

Received June 4, 2012; accepted after revision July 9

From the Division of Interventional Neuroradiology, Abbott Northwestern Hospital, Minneapolis, Minnesota.

No external sources of funding were used for the completion of this study. Paper previously presented in part at: International Stroke Conference, February 1-2, 2012; New Orleans, Louisiana; and in whole at: 50th Annual Meeting of the American Society of Neuroradiology and the Foundation of the ASNR Symposium, April 21-26, 2012; New York, New York.

Please address correspondence to Josser E. Delgado Almandoz, MD, Division of Interventional Neuroradiology, Abbott Northwestern Hospital, 800 E 28th St, Minneapolis, MN 55407, e-mail: josser.delgado@crlmed.com

三 Indicates article with supplemental on-line table.

http://dx.doi.org/10.3174/ajnr.A3291 mality as the cause of SAH despite negative initial neurovascular examinations is important because instituting treatment may avert rehemorrhage leading to increased morbidity and mortality. $^{3}$

In recent years, advances in CTA and MRA have allowed these techniques to become valuable noninvasive alternatives to DSA for the evaluation of patients with $\mathrm{SAH}$, with some institutions advocating CTA as the primary means of diagnosis in this patient population. ${ }^{29,32,33}$ However, DSA continues to be the criterion standard for the evaluation of patients with SAH and remains the primary means of diagnosis at many centers. ${ }^{34,35}$

Most prior studies have examined the yield of repeat DSA in patients with SAH who have a negative initial DSA study, which has varied widely from $0 \%$ to $36 \%{ }^{6,7,10-14,16-19,21-28,30,31}$ To date, only 1 study has examined the yield of DSA in patients with a negative initial CTA, which was $10 \%$ in patients with diffuse SAH, $17 \%$ in patients with peripheral sulcal SAH, and $0 \%$ in patients with perimesencephalic SAH. ${ }^{29}$

The present study aimed to determine the yield of DSA for the detection of causative vascular lesions in a cohort of patients with 

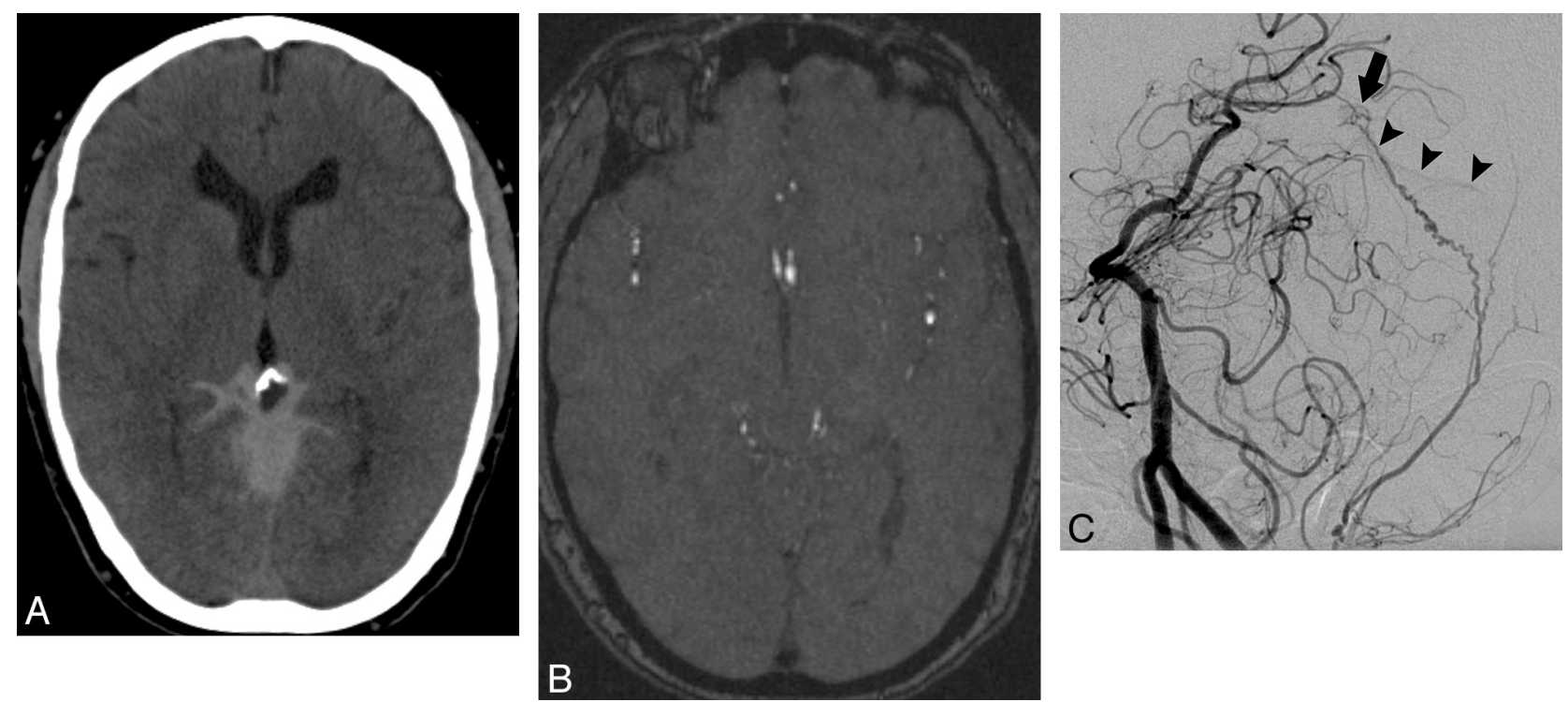

FIG 1. A 58-year-old woman with a known allergy to iodinated contrast material who presented with acute onset of severe headache. A, NCCT demonstrates a diffuse pattern of SAH centered in the posterior aspect of the basal cisterns with extension to the occipital and parietal sulci. $B$, MRA source image in the region of the hemorrhage does not demonstrate a causative vascular abnormality. $C$, Oblique left vertebral angiogram obtained 1 day after the MRA and following premedication with steroids demonstrates a small DAVF deriving arterial supply from an enlarged meningeal branch of the left vertebral artery (arrow) and with early venous drainage to the straight sinus (arrowheads). The DAVF was treated with radiosurgery.

SAH who had negative initial noninvasive neurovascular examinations (CTA or MRA).

\section{MATERIALS AND METHODS \\ Patient Selection and Study Protocol}

Our study was approved by the institutional review board of our hospital and was conducted in compliance with the Health Insurance Portability and Accountability Act. We conducted a retrospective review of prospectively collected data of patients who presented to our institution from January 1, 2007, until December 31,2011, with nontraumatic SAH evidenced by NCCT of the head or CSF xanthochromia and an initial CTA or MRA that was negative for a causative vascular abnormality. According to our institutional protocol, we performed DSA of the intracranial circulation within 24 hours of the initial noninvasive neurovascular examination; if the findings were negative, a repeat DSA examination was performed 7 days after presentation to identify a causative vascular etiology for the SAH.

\section{Image Acquisition}

NCCT acquisitions were performed according to standard protocols on 16- (LightSpeed) or 64-section helical CT scanners (Discovery HD; GE Healthcare, Milwaukee, Wisconsin). CTA acquisitions were performed on 16- (LightSpeed) or 64-section helical CT scanners (Discovery HD) by scanning from the base of the $\mathrm{C} 1$ vertebral body to the vertex by using an axial technique, 0.5 pitch, 0.625-mm collimation, 350 maximal mA, $120 \mathrm{kVp}$ (16-section) or 40 - to $140-\mathrm{kVp}$ spectral beam (64-section), 22-cm FOV, and $80-100 \mathrm{~mL}$ of iodinated contrast material administered by power injector at $4-5 \mathrm{~mL}$ per second into an antecubital vein by using SmartPrep (GE Healthcare), a semiautomated bolus tracking technique.
MRA examinations were performed on either 1.5T or 3T scanners (Symphony 1.5T, Skyra 3T, or Trio 3T; Siemens, Erlangen, Germany) by using standard arterial time-of-flight sequences provided by the vendor.

DSA was performed by using a dedicated biplanar neuroangiographic unit (Axiom Artis; Siemens) with transfemoral arterial access and intravenous conscious sedation. All DSA examinations included biplanar intracranial images after selective catheterization and contrast injection of both external carotid arteries and ICAs and at least 1 vertebral artery. Standard anteroposterior, lateral, and at least 2 oblique views of each vessel injected were obtained as part of our routine imaging protocol. In cases in which reflux opacification of the contralateral vertebral artery and PICA was not achieved from a given vertebral artery injection, we catheterized the contralateral vertebral or subclavian artery for imaging of the ipsilateral vertebral artery and PICA. Rotational angiography with $3 \mathrm{D}$ reconstructions was performed at the discretion of the interventional neuroradiologist but is not part of the routine imaging protocol at our institution.

\section{Image Analysis}

The NCCT examinations were independently reviewed by 2 experienced interventional neuroradiologists to determine the Fisher grade and pattern of SAH, categorized as diffuse, perimesencephalic, or peripheral sulcal. Perimesencephalic SAH was defined as SAH located mainly within the interpeduncular, prepontine, premedullary, ambient, and/or quadrigeminal plate cisterns without extension to the ventricular system, as defined by van Gijn et al. ${ }^{36}$

The DSA examinations were reviewed by 2 experienced interventional neuroradiologists to determine the presence of a caus- 

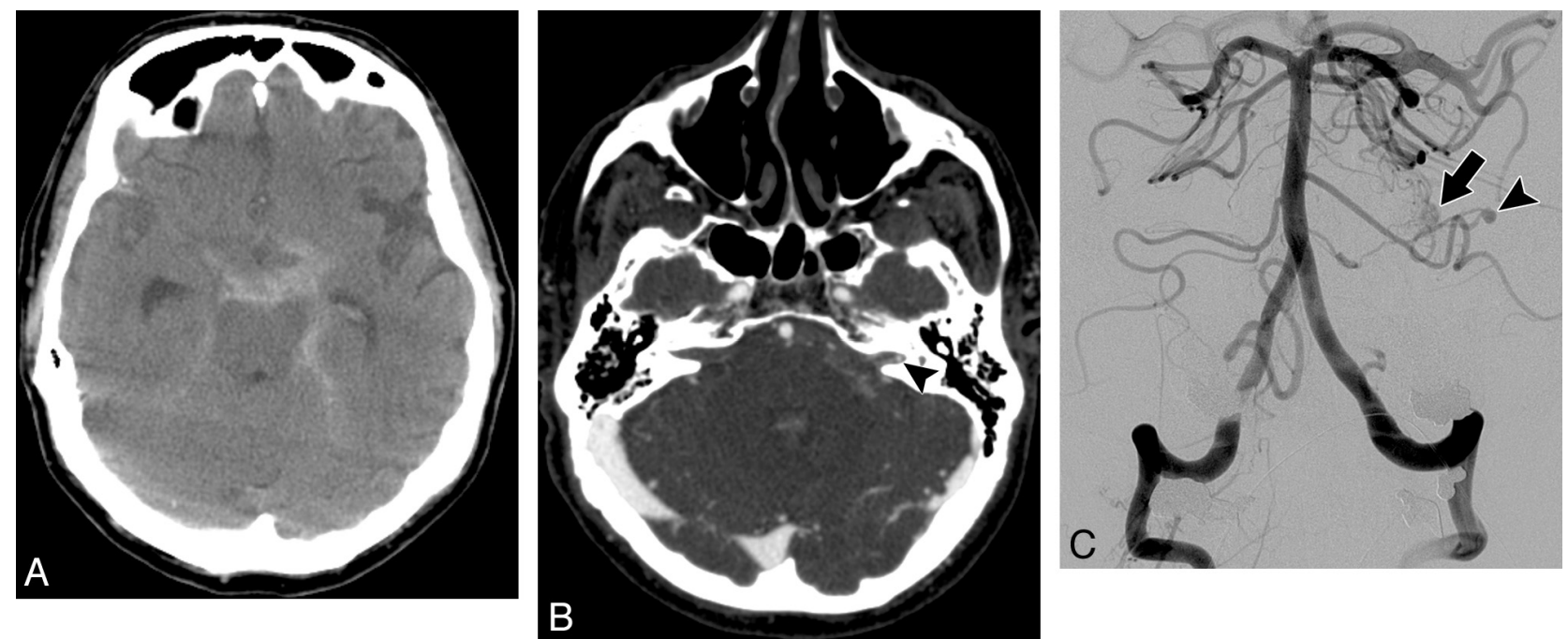

FIG 2. A 71-year-old man who presented with the worst headache of his life. A, NCCT demonstrates a diffuse pattern of SAH in the basal cisterns. B, Axial CTA source image was initially interpreted as not demonstrating a causative vascular abnormality for the hemorrhage. In retrospect, a small aneurysm is present in the lateral aspect of the left internal auditory canal (arrowhead). C, Frontal right vertebral angiogram obtained 1 day after the CTA demonstrates a small pial AVM in the region of the left cerebellopontine angle (arrow) with an associated 2-mm feeding artery aneurysm from a distal branch of an enlarged left anterior inferior cerebellar artery (arrowhead). The patient underwent aneurysm coiling followed by radiosurgery of the AVM nidus.
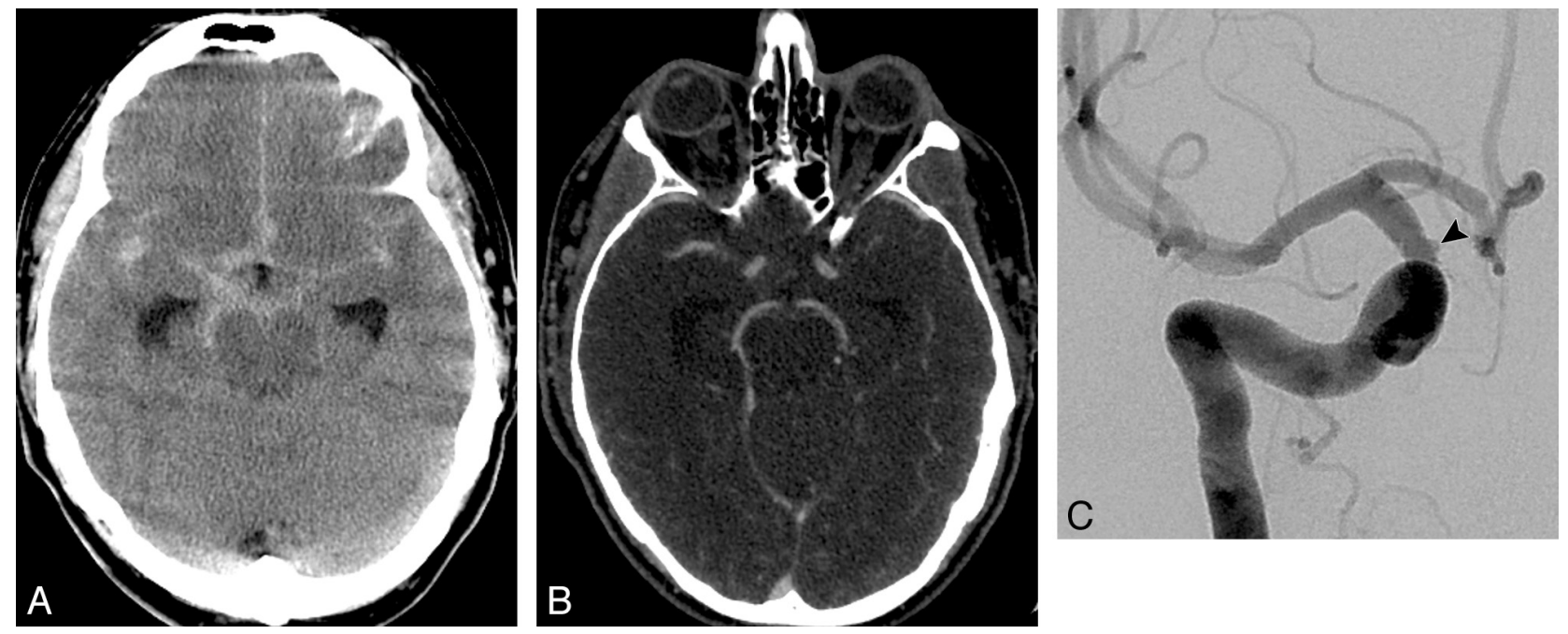

FIG 3. A 50 -year-old man who presented with sudden onset of severe headache, nausea, and vomiting. $A$, NCCT demonstrates a diffuse pattern of SAH with extension to the Sylvian fissures. B, Axial CTA source image did not demonstrate a causative vascular abnormality. C, Oblique right internal carotid angiogram obtained 1 day after the CTA demonstrates a 1.5-mm blister right supraclinoid ICA aneurysm. The patient underwent endovascular aneurysm treatment with the Pipeline Embolization Device (Covidien/ev3 Neurovascular, Irvine, California).

ative vascular abnormality for the SAH. Differences in reader interpretation for the SAH pattern in the NCCT and the presence of a causative vascular abnormality in the catheter angiograms were adjudicated by consensus. Treatment decisions were reached by consensus of a panel comprising experienced interventional neuroradiologists and neurologic surgeons.

\section{Medical Record Review}

Medical records were reviewed for patient age, sex, family history of cerebral aneurysms, smoking history, admission Hunt and Hess scale, length of intensive care unit stay, and length of hospital stay.

\section{Statistical Analysis}

Statistical analysis was performed using the MedCalc, Version 11.1, software package (MedCalc Software, Mariakerke, Belgium). Interobserver agreement for the $\mathrm{SAH}$-pattern categorization was determined with the $\kappa$ statistic. A $P$ value $\leq .05$ was considered statistically significant.

\section{RESULTS}

From January 1, 2007, until December 31, 2011, a total of 302 patients presented to our institution with nontraumatic SAH. Of these, 55 patients underwent an initial noninvasive neurovascular examination that was negative for a causative vascular abnormality (18.2\%), comprising the patient cohort of this study. The ini- 
tial examination was a CTA in 47 patients $(85.5 \%)$ and an MRA in 8 patients (14.5\%). Mean patient age was 58.2 years (median, 58 years; range, 25-88 years). Twenty-eight patients were men (50.9\%), and 27 were women $(49.1 \%)$. The mean length of intensive care unit stay was 7.7 days (median, 5 days; range, $0-34$ days). The mean length of the hospital stay was 11.3 days (median, 9 days; range, 1-34 days). The On-line Table summarizes the clinical and radiologic characteristics of the patient cohort.

Thirty-three patients had diffuse $\mathrm{SAH}$ (60\%), 11 patients had perimesencephalic SAH (20\%), and 11 patients had peripheral sulcal SAH (20\%). No patients with CSF xanthochromia were present in our cohort. Interobserver agreement for the categorization of SAH on the NCCT was excellent ( $\kappa=0.86$; 95\% CI, $0.84-0.88$ ). Of note, 6 of the 8 MRAs (75\%) were performed in patients presenting with peripheral sulcal $\mathrm{SAH}$, and 1 patient with diffuse SAH had an MRA performed first because of a known allergy to iodinated contrast material requiring premedication.

The mean time interval between the initial noninvasive neurovascular examination and the first DSA examination was 0.9 days (median, 1 day; range, $0-7$ days). The first DSA examination demonstrated a causative vascular lesion for the SAH in 5 patients (9.1\%), 4 of whom had diffuse SAH (yield of $12.1 \%$ ) and 1 of whom had peripheral sulcal SAH (yield of 9.1\%). The vascular lesions identified were 2 DAVFs (Fig 1), 1 pial cerebellopontine angle AVM with a 2-mm feeding artery aneurysm located in the internal auditory canal (Fig 2), a 2-mm blister ICA aneurysm (Fig 3), and a case of vasculopathy due to reversible cerebral vasoconstriction syndrome in a patient with peripheral sulcal SAH (Fig 4). One of the DAVFs and the blister ICA aneurysm were treated with endovascular embolization; the other DAVF was treated with radiosurgery alone. The pial AVM was treated with endovascular embolization of the feeding artery aneurysm followed by radiosurgery of the AVM nidus, and the case of vasculopathy was treated with cessation of the inciting medication. No causative vascular lesions were found in patients with perimesencephalic SAH in the first DSA examination.

A second DSA examination was performed in 32 patients (58.2\%), including 23 of the 29 patients with diffuse SAH who had a negative first DSA examination (79.3\%), 7 of the 11 patients with perimesencephalic SAH (63.6\%), and 2 of the 10 patients with peripheral sulcal SAH who had a negative first DSA exami-
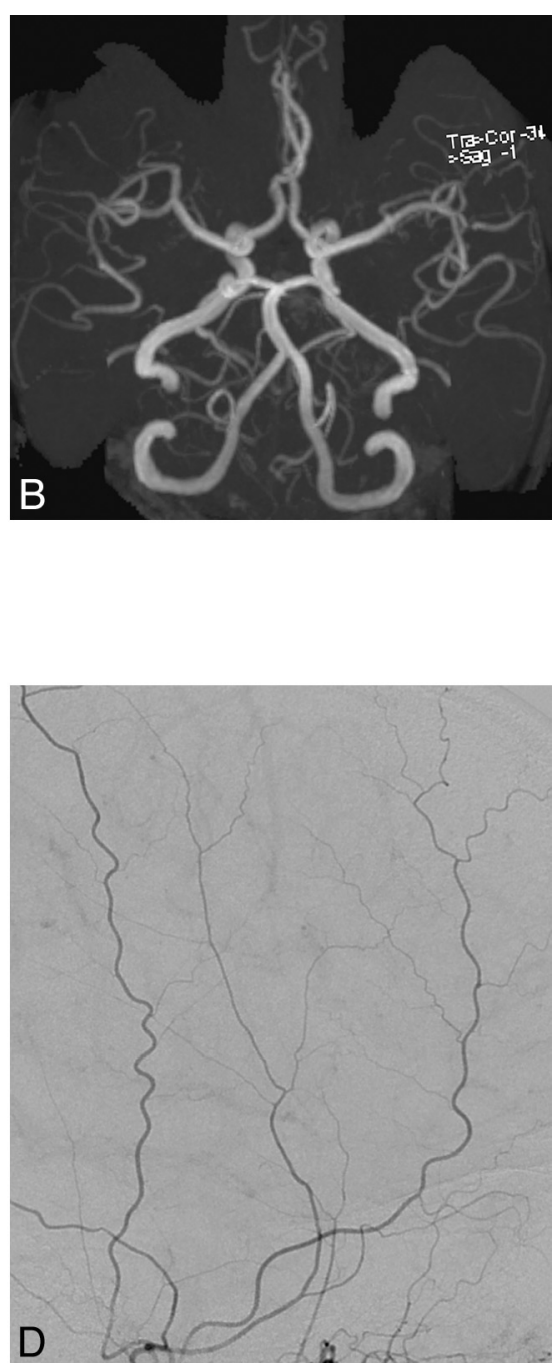

FIG 4. A 52-year-old woman with a history of migraine headaches who presented with sudden onset of severe frontal headache. A, NCCT demonstrates a peripheral sulcal pattern of SAH in frontal region. $B$, Axial MRA maximum-intensity-projection image does not demonstrate a causative vascular abnormality. $C$, Lateral right external carotid angiogram obtained 2 作 affecting the right superficial temporal artery (arrowheads), consistent with vasculopathy. The clinical scenario was consistent with reversible cerebral vasoconstriction syndrome. $D$, Lateral right external carotid artery angiogram obtained 3 months after cessation of the inciting medication demonstrates resolution of the abnormalities in the right superficial temporal artery.

nation (20\%). The mean time interval between the initial noninvasive neurovascular examination and the second DSA examination was 12.1 days (median, 9 days; range, 4-69 days). The second DSA examination demonstrated a causative vascular abnormality in 1 patient with diffuse SAH (yield of $4.3 \%$ ). This lesion was a $2-\mathrm{mm}$ anterior communicating artery aneurysm, which was treated with endovascular embolization (Fig 5). No causative vascular lesions were found in patients with perimesencephalic or peripheral sulcal SAH in the second DSA examination.

A third DSA examination was performed in 5 patients (9.1\%), all with diffuse SAH. The mean time interval between the initial noninvasive neurovascular examination and the third DSA examination was 29.4 days (median, 32 days; range, 12-53 days). No causative vascular lesions were found in the third DSA examination.

Overall, DSA demonstrated a causative vascular lesion for the 

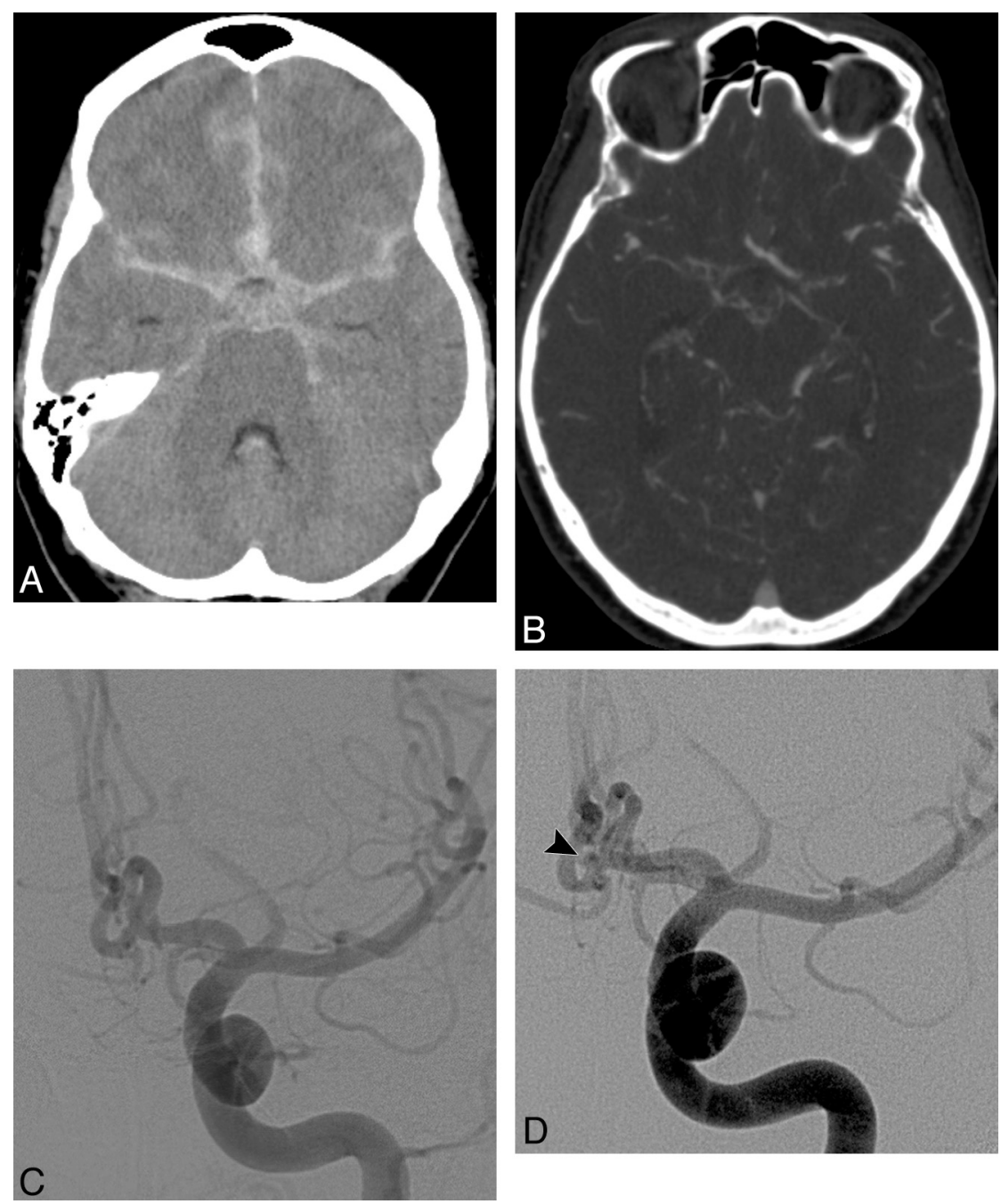

FIG 5. A 67-year-old woman who presented with sudden onset of severe headache. A, NCCT demonstrates a diffuse pattern of SAH with extension to the Sylvian fissures and ventricular system. B, Axial CTA source image did not demonstrate a causative vascular abnormality. $C$, Frontal left internal carotid angiogram obtained on the same day as the CTA did not demonstrate a causative vascular abnormality. $D$, Frontal left internal carotid artery angiogram obtained 8 days after the initial CTA demonstrates a 2-mm anterior communicating artery aneurysm (arrowhead). The patient underwent aneurysm coiling.

SAH in 6 patients (yield of 10.9\%), 5 of whom had diffuse SAH (yield of 15.2\%) and 1 of whom had peripheral sulcal SAH (yield of $9.1 \%$ ). No causative vascular lesions were found in patients with perimesencephalic SAH.

Retrospective review of the initial CTA and MRA showed that in 1 patient, the causative vascular lesion was evident but not recognized by the initial reader, and in the other 5 patients, the vascular lesion was not evident.

\section{DISCUSSION}

The frequency of negative initial CTA and MRA in patients with SAH in our study, $18.2 \%$, is similar to that reported by Agid et $\mathrm{al}^{29}$ in patients with SAH initially evaluated with CTA (22.1\%). These findings suggest that though CTA is a very useful initial noninvasive diagnostic tool for the evaluation of this patient population, there are still a significant minority of patients who will require further evaluation with DSA due to a negative initial noninvasive neurovascular examination.
We found that the overall yield of DSA in patients with SAH and negative initial neurovascular examinations was $10.9 \%$, with a higher yield in patients with diffuse (15.2\%) and peripheral (9.1\%) SAH compared with patients with perimesencephalic SAH (0\%). The overall yield of our study is within the range of past studies examining the yield of repeat DSA in patients with $\mathrm{SAH}$ and a negative initial DSA examination $\left(0 \%-14 \%{ }^{17,18,27,30,31}\right)$, but it is higher than that in the previous study by Agid et al examining the yield of DSA in patients with SAH and a negative initial CTA (4.2\%). The difference in overall yield of DSA between our study and that of Agid et $\mathrm{al}^{29}$ is likely explained by the larger proportion of patients with perimesencephalic SAH in their patient cohort $(48.2 \%)$ compared with that in our study (20\%). Indeed, the yield of DSA in the different SAH patterns is similar in the study of Agid et al and our study: 10\% and $15 \%$ for diffuse $\mathrm{SAH}, 17 \%$ and $9 \%$ for peripheral sulcal SAH, and $0 \%$ for perimesencephalic SAH in both studies. Similarly, Cruz et $\mathrm{al}^{33}$ also found the yield of DSA in a cohort of 43 patients with perimesencephalic SAH and a negative initial CTA to be $0 \%$. These results suggest that performing conventional angiography after a negative CTA may not be necessary in patients with perimesencephalic SAH. However, a recent study by Delgado Almandoz et $\mathrm{al}^{30}$ reported finding a causative cerebral aneurysm at repeat DSA performed 7 days after presentation in 1 of 29 patients with perimesencephalic SAH who had negative initial DSA and CTA examinations (yield of $3.4 \%$ ). The combined sample size of patients with perimesencephalic SAH and negative initial neurovascular examinations in these 4 studies is modest (176 patients), and future prospective studies with larger sample sizes may be needed to definitively determine when to stop imaging this specific patient population.

In our study, a second DSA examination performed 7 days after the initial noninvasive examination yielded positive results only in patients with diffuse SAH (yield of $4.3 \%$ ), which was similar to that reported by Delgado Almandoz et al ${ }^{30}$ (yield of 5.1\%) but lower than that reported by Maslehaty et $\mathrm{al}^{31}$ (yield of 10.8\%) in patients with nonperimesencephalic SAH. In both the present study and that of Delgado Almandoz et al, a third DSA examination did not reveal any additional underlying neurovascular abnormalities. These results suggest that patients with diffuse $\mathrm{SAH}$ would benefit from a second DSA examination performed approximately 7 days after the initial neurovascular examination but not from a third DSA examination thereafter. 
We found that the causative vascular abnormalities demonstrated by DSA in our cohort were a combination of DAVFs, small pial AVMs, small aneurysms, and vasculopathy, which were not preferentially localized to any particular blood vessel or region of the skull base. This is in contradistinction to the previous study by Agid et al, ${ }^{29}$ in which small aneurysms and vasculopathy encompassed all of the vascular lesions identified by DSA but no DAVFs or AVMs were found. Our results suggest that detailed evaluation of the entire neurovascular tree, including the external carotid artery branches, is important to confidently exclude a vascular lesion as the SAH etiology in patients with negative initial noninvasive neurovascular examinations.

The limitations of our study are the lack of rotational angiography with $3 \mathrm{D}$ reconstructions in all catheter angiograms and the modest sample size.

\section{CONCLUSIONS}

DSA is a valuable tool in the evaluation of patients with diffuse and peripheral sulcal SAH who have a negative initial noninvasive neurovascular examination, demonstrating a causative vascular lesion in $15.2 \%$ and $9.1 \%$ of patients, respectively.

\section{ACKNOWLEDGMENTS}

We acknowledge Sandee K. Verootis, Radiology Department, Abbott Northwestern Hospital, for her contribution in the data collection for this study.

Disclosures: Josser E. Delgado Almandoz-UNRELATED: Travel/Accommodations/ Meeting Expenses Unrelated to Activities Listed: Covidien/ev3. Benjamin M. Crandell_UNRELATED: Payment for Lectures (including service on Speakers Bureaus): ev3, Comments: balloon-remodeling techniques course. David E. TubmanUNRELATED: Consultancy: Covidien/ev3, MicroVention, Lake Region Medical, Payment for Lectures (including service on Speakers Bureaus): Covidien/ev3.

\section{REFERENCES}

1. King JT Jr. Epidemiology of aneurysmal subarachnoid hemorrhage. Neuroimaging Clin N Am 1997;7:659-68

2. Hijdra A, Braakman R, van Gijn J, et al. Aneurysmal subarachnoid hemorrhage: complications and outcome in a hospital population. Stroke 1987;18:1061-67

3. Hijdra A, van Gijn J, Nagelkerke NJ, et al. Prediction of delayed cerebral ischemia, rebleeding, and outcome after aneurysmal subarachnoid hemorrhage. Stroke 1988;19:1250-56

4. van Gijn J, Rinkel GJ. Subarachnoid haemorrhage: diagnosis, causes and management. Brain 2001;124:249-78

5. Hop JW, Rinkel GJ, Algra A, et al. Changes in functional outcome and quality of life in patients and caregivers after aneurysmal subarachnoid hemorrhage. J Neurosurg 2001;95:957-63

6. Nishioka H, Torner JC, Graf CJ, et al. Cooperative study of intracranial aneurysms and subarachnoid hemorrhage: a long-term prognostic study. III. Subarachnoid hemorrhage of undetermined etiology. Arch Neurol 1984;41:1147-51

7. Suzuki S, Kayama T, Sakurai Y, et al. Subarachnoid hemorrhage of unknown cause. Neurosurgery 1987;21:310-13

8. Farrés MT, Ferraz-Leite H, Schindler E, et al. Spontaneous subarachnoid hemorrhage with negative angiography: CT findings. J Comput Assist Tomogr 1992;16:534-37

9. Ferbert A, Hubo I, Biniek R. Non-traumatic subarachnoid hemorrhage with normal angiogram: long-term follow-up and CT predictors of complications. J Neurol Sci 1992;107:14-18

10. Pathirana N, Refsum SE, McKinstry CS, et al. The value of repeat cerebral angiography in subarachnoid haemorrhage. BrJ Neurosurg 1994;8:141-46
11. Duong $\mathrm{H}$, Melancon $\mathrm{D}$, Tampieri $\mathrm{D}$, et al. The negative angiogram in subarachnoid haemorrhage. Neuroradiology 1996;38:15-19

12. Bradac GB, Bergui M, Ferrio MF, et al. False-negative angiograms in subarachnoid haemorrhage due to intracranial aneurysms. Neuroradiology 1997;39:772-76

13. du Mesnil de Rochemont R, Heindel W, Wesselmann C, et al. Nontraumatic subarachnoid hemorrhage: value of repeat angiography. Radiology 1997;202:798-800

14. Urbach $H$, Zentner J, Solymosi $L$. The need for repeat angiography in subarachnoid haemorrhage. Neuroradiology 1998;40:6-10

15. Hashimoto H, Iida J, Hironaka $Y$, et al. Use of spiral computerized tomography angiography in patients with subarachnoid hemorrhage in whom subtraction angiography did not reveal cerebral aneurysms. J Neurosurg 2000;92:278-83

16. Inamasu J, Nakamura Y, Saito R, et al. “Occult” ruptured cerebral aneurysms revealed by repeat angiography: result from a large retrospective study. Clin Neurol Neurosurg 2003;106:33-37

17. Topcuoglu MA, Ogilvy CS, Carter BS, et al. Subarachnoid hemorrhage without evident cause on initial angiography studies: diagnostic yield of subsequent angiography and other neuroimaging tests. J Neurosurg 2003;98:1235-40

18. Andaluz N, Zuccarello M. Yield of further diagnostic work-up of cryptogenic subarachnoid hemorrhage based on bleeding patterns on computed tomographic scans. Neurosurgery 2008;62:1040-46

19. van Rooij WJ, Peluso JP, Sluzewski M, et al. Additional value of $3 \mathrm{D}$ rotational angiography in angiographically negative aneurysmal subarachnoid hemorrhage: how negative is negative? AJNR Am J Neuroradiol 2008;29:962-66

20. Maslehaty $H$, Petridis AK, Barth $H$, et al. Diagnostic value of magnetic resonance imaging in perimesencephalic and nonperimesencephalic subarachnoid hemorrhage of unknown origin. J Neurosurg 2011;114:1003-07

21. Forster DM, Steiner L, Hakanson S, et al. The value of repeat panangiography in cases of unexplained subarachnoid hemorrhage. J Neurosurg 1978;48:712-16

22. Gilbert JW, Lee C, Young B. Repeat cerebral pan-angiography in subarachnoid hemorrhage of unknown etiology. Surg Neurol 1990; 33:19-21

23. Iwanaga H, Wakai S, Ochiai C, et al. Ruptured cerebral aneurysms missed by initial angiographic study. Neurosurgery 1990; 27:45-51

24. Kaim A, Proske M, Kirsch E, et al. Value of repeat-angiography in cases of unexplained subarachnoid hemorrhage (SAH). Acta Neurol Scand 1996;93:366-73

25. Huttner HB, Hartmann M, Kohrmann M, et al. Repeated digital substraction angiography after perimesencephalic subarachnoid hemorrhage? J Neuroradiol 2006;33:87-89

26. Jung JY, Kim YB, Lee JW, et al. Spontaneous subarachnoid haemorrhage with negative initial angiography: a review of 143 cases. J Clin Neurosci 2006;13:1011-17

27. Little AS, Garrett M, Germain R, et al. Evaluation of patients with spontaneous subarachnoid hemorrhage and negative angiography. Neurosurgery 2007;61:1139-50

28. Ishihara H, Kato S, Akimura T, et al. Angiogram-negative subarachnoid hemorrhage in the era of three dimensional rotational angiography. J Clin Neurosci 2007;14:252-55

29. Agid R, Andersson T, Almqvist H, et al. Negative CT angiography findings in patients with spontaneous subarachnoid hemorrhage: when is digital subtraction angiography still needed? AJNR Am J Neuroradiol 2010;31:696-705

30. Delgado Almandoz JE, Jagadeesan BD, Refai D, et al. Diagnostic yield of repeat catheter angiography in patients with catheter and computed tomography angiography negative subarachnoid hemorrhage. Neurosurgery 2012;70:1135-42

31. Maslehaty H, Barth H, Petridis AK, et al. Special features of subarachnoid hemorrhage of unknown origin: a review of a series of 179 cases. Neurol Res 2012;34:91-97

32. Fox AJ, Symons SP, Aviv RI. CT angiography is state-of-the-art first 
vascular imaging for subarachnoid hemorrhage. AJNR Am J Neuroradiol. 2008;29:e41-42, author reply e46-47

33. Cruz JP, Sarma D, Noel de Tilly L. Perimesencephalic subarachnoid hemorrhage: when to stop imaging? Emerg Radiol 2011;18: 197-202

34. Kallmes DF, Layton K, Marx WF, et al. Death by nondiagnosis: why emergent $\mathrm{CT}$ angiography should not be done for patients with subarachnoid hemorrhage. AJNR Am J Neuroradiol 2007;28: 1837-38

35. Moran CJ. Aneurysmal subarachnoid hemorrhage: DSA versus CT angiography-is the answer available? Radiology 2011;258:15-17

36. van Gijn J, van Dongen KJ, Vermeulen M, et al. Perimesencephalic hemorrhage: a nonaneurysmal and benign form of subarachnoid hemorrhage. Neurology 1985;35:493-97 